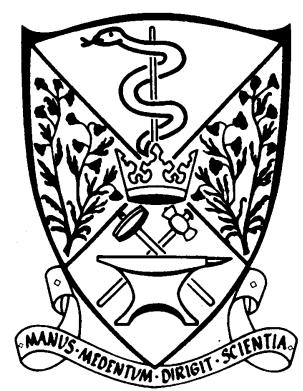

Department of Medicine, Hammersmith Hospital, London W12 0HS Case presented by: Helen Fidler, MB

Radiology:

A Booth, FRCR, senior registrar

Chairman:

H J F Hodgson, FRCP, reader in medicine

Discussion group:

J Calam, FRCP, senior lecturer in gastroenterology

L Luzatto, FRCP, professor of haematology

J M B Hughes, FRCP, reader

in respiratory medicine

Series edited by: Dr Robert Winter.

BrMed F 1990;300:590-2

\title{
Portal vein thrombosis in myeloproliferative disease
}

\author{
Uses of thrombolytic and antiplatelet treatment
}

Portal vein thrombosis may be the first presentation of a hypercoagulable state. We report a case that illustrates a diagnostic approach to show the extent of the splanchnic venous thrombus and to investigate its haematological aetiology. The discussion focused on the association between portal vein thrombosis and myeloproliferative disease and controversies in managing this condition.

\section{Case history}

A 59 year old woman was admitted to hospital with central abdominal pain, nausea, and copious vomiting that had persisted for 48 hours. She had experienced a similar but less severe episode one month previously but had no other history. On examination she was febrile and there were no stigmata of chronic liver disease. Her spleen was noticeably enlarged to $15 \mathrm{~cm}$ below the costal margin. No ascites was present. Investigation showed her haemoglobin concentration to be $180 \mathrm{~g} / \mathrm{l}$, her white blood cell count to be $21 \cdot 2 \times$ $10^{9} / 1(88 \%$ neutrophils), and her platelet count to be $600 \times 10^{9} / 1$. Blood concentrations of urea and electrolytes and serum activity of amylase were normal and liver function tests gave normal results. The concentration of $\mathrm{C}$ reactive protein was $46 \mathrm{mg} / \mathrm{l}$ (normal range $<10 \mathrm{mg} / \mathrm{l}$ ). Doppler ultrasonography at the referring hospital confirmed pronounced splenomegaly and showed portal, splenic, and superior mesenteric vein thrombosis with normal echogenicity over the liver. Initial management consisted of full heparinisation, opiate analgesia, and venesection.

She was referred for further evaluation. There were no new findings on examination. Her blood count (after venesection) showed a haemoglobin concentration of $154 \mathrm{~g} / 1$, a white cell count of $14 \times 10^{9} / 1$, and a platelet count of $390 \times 10^{9} / 1$. Further investigations were directed at showing the extent of the thrombosis and the nature of the haematological disorder.

Doppler ultrasonography (figs 1 and 2) showed increased echogenicity in the right and left intrahepatic and main extrahepatic veins with areas of no noticeable flow. The splenic and superior mesenteric veins also showed similar appearances. Visceral angiography (fig 3) and an indirect portogram confirmed this and showed the spleen to be massively enlarged. Several wedge shaped splenic infarcts were seen and numerous collateral veins were evident.

Computed tomography (fig 4) confirmed splenomegaly and collateral vessels at the splenic hilus. The portal vein, splenic vein, and superior mesenteric vein to the level of the iliac crest showed central low attenuation with an enhancing outer rim indicative of extensive thrombosis. In summary, the radiological investigations showed widespread thrombosis of the extrahepatic portal venous system with splenomegaly and several splenic infarcts. Upper gastrointestinal endoscopy showed moderate oesophageal varices and multiple shallow duodenal ulcers.

The following further investigations gave normal results: Ham and sucrose lysis test, autoantibody screening, anticardiolipin antibody activities (IgG and IgM), cold agglutinin concentrations, direct antiglobulin test, and antithrombin $\mathrm{C}$ and protein $\mathrm{S}$ concentrations. Neutrophil alkaline phosphatase activity was raised, being 219 units/100 neutrophils (normal range 35-100), which taken with the raised white cell count and haemoglobin value, strongly suggested a primary myeloproliferative disorder. A

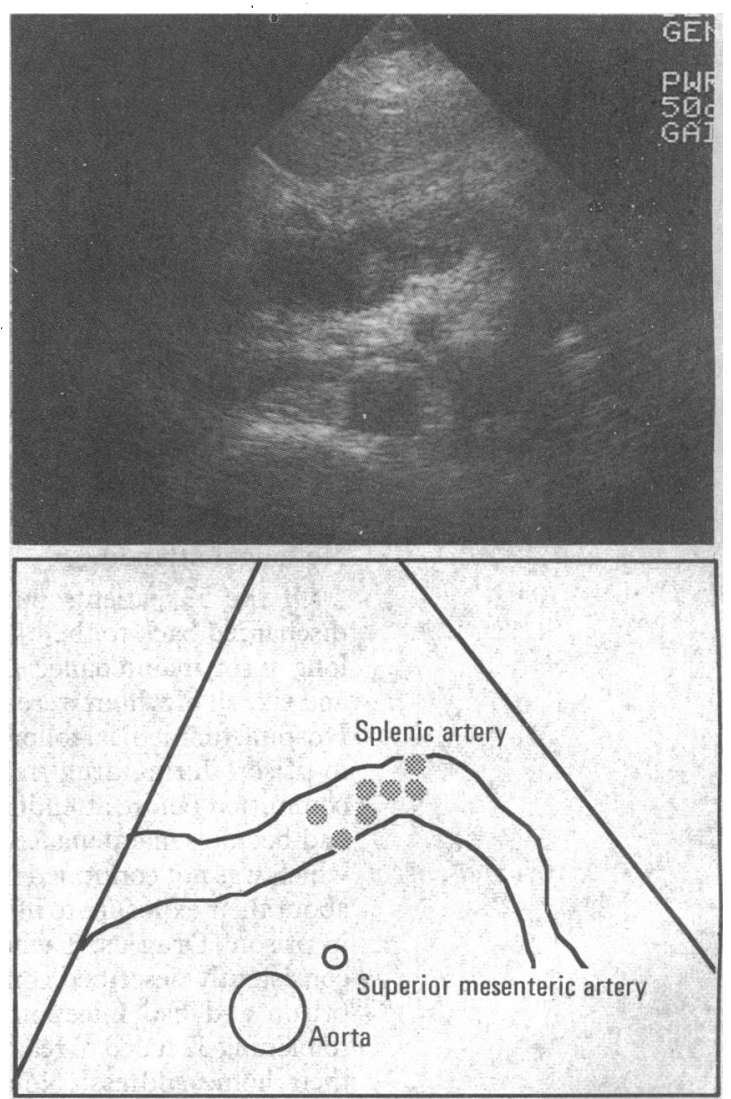

FIG 1-Top: Two dimensional ultrasonogram showing echogenic material within the splenic vein; appearances of those of extensive thrombus. Bottom: Diagram of ultrasonogram

trephine bone marrow biopsy specimen was noticeably hypercellular, with an increase in all cell lines but especially in megakaryocytes, which were clustered and dysplastic. Full heparinisation was continued for a further week and warfarin treatment was started. Although thrombolytic treatment can be effective in managing portal vein thrombosis (see comment below), the presence of duodenal ulcer disease at endoscopy precluded its use. Over the next three weeks all clinical symptoms resolved and she was discharged for haematological outpatient follow up.

\section{Comment}

Although the association between myeloproliferative disorders such as polycythaemia rubra vera, essential thrombocythaemia, myelofibrosis and chronic myeloid leukaemia, and hypercoagulable states is well recognised, the incidence of myeloproliferative disorders in patients presenting with splanchnic vein thrombosis may be higher than generally appreciated. In a recent study of adult patients presenting with portal vein thrombosis an underlying myeloprolifera- 


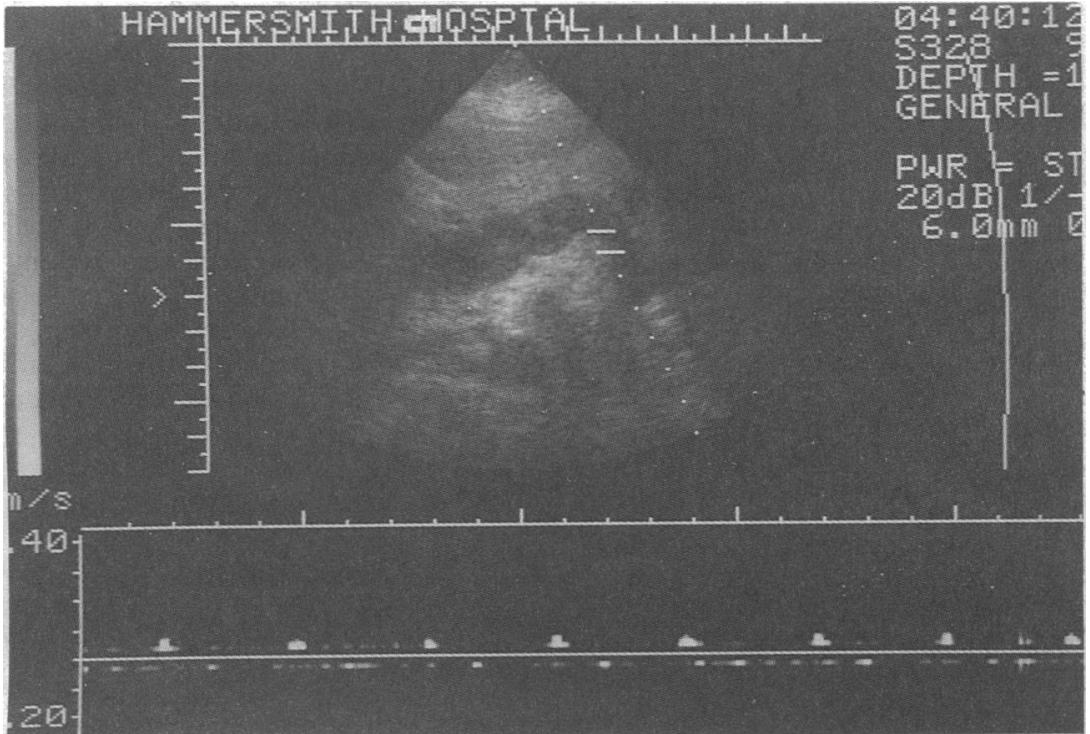

FIG 2-Doppler

ultrasonography. As for figure but with Doppler probe sited in splenic vein. Lower panel shows no Doppler signal, indicating no flow within vessel

tive disease was diagnosed in almost half of them.' In this prospective series of 33 patients nine had polymia. In only half of them, however, would the cythaemia rubra vera and six essential thrombocythaecondition have been diagnosed at presentation from the usual criteria of suggestive full blood count, increased red cell mass, and compatible bone marrow histology. In four patients the diagnosis became apparent only during extended observation at follow up. In three the evidence of a myeloproliferative disorder was the production of erythroid colonies when bone marrow progenitor cells were cultured without added erythropoietin. The results of this study suggested that primary myeloproliferative disorders, in a full blown or at an early stage, are an important cause of splanchnic vein thrombosis. The mechanism of thrombosis in myeloproliferative diseases is not likely to be due to changes in blood viscosity alone: thrombosis may occur when the packed cell volume is normal, and platelet aggregation studies point to concurrent platelet dysfunction.

Rapid radiological diagnosis of splenoportal and superior mesenteric vein thrombosis has permitted the development of non-surgical approaches to treatment. Verbanck et al report on a patient with partial portal, splenic, and superior mesenteric obstruction and underlying essential thrombocythaemia. ${ }^{3}$ Treatment with continuous heparin infusion, dipyridamole, and aspirin resulted in reduction of abdominal pain and partial recanalisation of splenoportal and superior mesenteric veins after three weeks. After seven weeks of treatment there was filling of the portal vein and its radicles with contrast material.

The role of thrombolysis in the treatment of

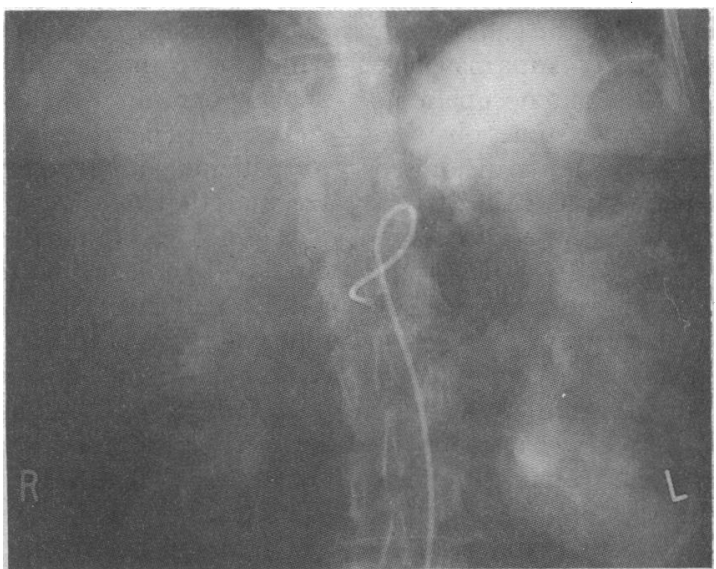

FIG 3-Late stage angiogram showing an enlarged spleen with several wedge shaped infarcts (sidewinder catheter seen in splenic artery) splanchnic vein thrombosis is unclear. Robin et al reported on a 20 year old man who presented with epigastric and left hypochondrial pain and was found to have superior mesenteric and splenic vein thrombosis. ${ }^{+}$Recombinant tissue plasminogen activator was given on the second and third days after presentation, and angiography after seven days showed complete recanalisation of the affected vessels. Currently, however, insufficient information is available to decide which of the above two options for medical management is the more effective or what the role of long term anticoagulation and antiplatelet treatment is. The risk of gastrointestinal bleeding in such predisposed patients should not be underestimated, and endoscopy is almost invariably indicated.

\section{Discussion}

JC: This patient was referred for consideration of thrombolytic treatment, but because of several relative risk factors including duodenal ulcer disease, the presence of varices, and a history of pain going back one month - which were all felt to alter the ratio of risk to benefit adversely - this was not given.

HJFH: One theoretical advantage is that tissue plasminogen activator might be expected to induce bleeding in an area of recent clotting but not in areas that had not bled recently. Do you think that tissue plasminogen activator might, in fact, have carried less risk than heparin?

LL: There has been no systematic comparative study of the relative risks of bleeding complications with heparin and with thrombolytic agents. I think that if duodenal ulcers were seen at endoscopy they may have bled to a small degree, even though this was not clinically apparent. Therefore I think that the decision not to use tissue plasminogen activator was perfectly reasonable. A practical advantage of heparin is its short half life and the relative ease with which its actions can be reversed in the event of bleeding.

Turning to the haematological disorder, the assumption that she has a myeloproliferative disorder is I am sure correct: there was no other reason for her to have a haemoglobin concentration of $180 \mathrm{~g} / \mathrm{l}$; her oxygen saturation and lung function were normal and, of course, her platelet count was also greatly raised. Her raised neutrophil alkaline phosphatase activity provides useful corroborative evidence. In brief, she met all the classic A and B criteria of the Polycythaemia Vera Study Group. The difficulty in managing patients with polycythaemia rubra vera is that they can have either thrombotic or haemorrhagic manifestations, and sometimes both. In this particular case the thrombotic tendency was likely to be due to not just quantitative but also qualitative platelet abnormalities. Several

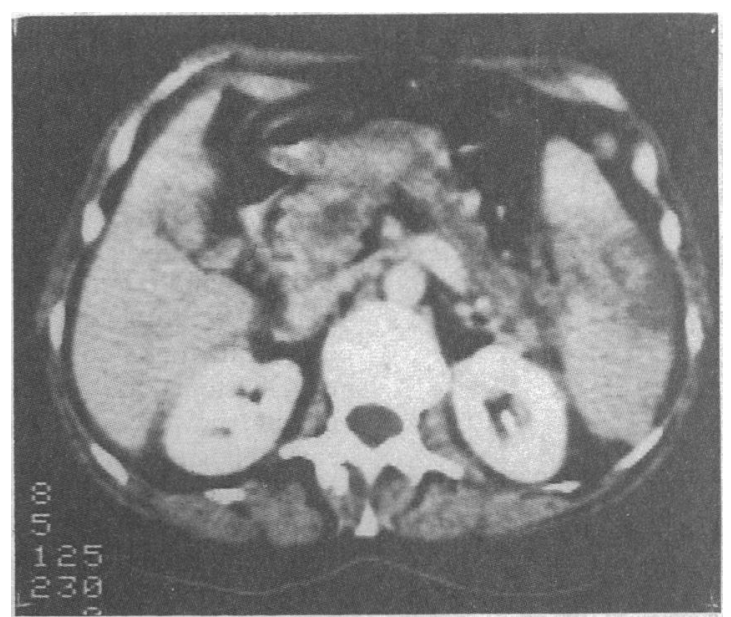

FIG 4-Computed tomogram showing peripheral infarcts in spleen and thrombus in splenic and portal veins 
studies suggest that platelet aggregation is increased in polycythaemia rubra vera. Despite reservations about the applicability of these ex vivo tests of platelet function, it may be that even when the platelet count has returned to normal-for example, with hydroxyurea - the functional platelet abnormality may persist, giving continued risks of thrombotic complications. I think that in such patients there is a case for using aspirin, which is probably the best antiplatelet drug available.

HJFH: Would you advocate continuing her treatment with warfarin?

LL: That is a difficult question. I think the answer must be yes, at least initially, because while large thrombi are still present they could form a focus for recurrence or extension. I think that it would be reasonable to consider withdrawing warfarin after six months, but in this decision you would need to be guided by the ultrasonic appearances of the splanchnic venous system and the calculated flows within it.

JMBH: Why should her portal vein be thrombosed? Is there any evidence for hepatic disease here?
HJFH: We have seen that the liver function tests and ultrasonography of the upper abdomen gave normal results and that there was no indication for a biopsy. The portal vein carries blood at a low flow rate between two venous circulations, making it particularly susceptible when there is a hypercoagulable state. Interestingly, studies are now showing a much higher incidence of haematological disease in patients presenting with splanchnic vein thrombosis. Given these observations, I think that good clinical management of such problems must now include careful haematological investigation at presentation and longer follow up.

1 Valla D, Casadevall N, Huisse MG, et al. Etiology of portal vein thrombosis in aduls. A prospective evaluation of primary myeloproliferative disorders. adults. A prospective evaluation

2 Schafer AI. Bleeding and thrombosis in the myeloproliferative disorders. Blood 1984;64:1-12.

3 Verbanck JJ, Rutgeerts LT, Haerens $M H$, et al. Partial splenoportal and superior. mesenteric vein thrombosis. Early sonographic diagnosis and successful conservative management. Gastroenterology 1984;86:949-52.

4 Robin P, Gruel Y, Lang M, Lagarrigue F, Scotto JM. Complete thrombolysis of mesenteric vein occlusion with recombinant tissue-type plasminogen activator. Lancet 1988;i:1391.

\section{New Drugs}

\section{Gall stones}

\section{Ian A D Bouchier}

In the 100 years after the first operation on the gall bladder by John Stough Bobbs cholecystectomy became the accepted method of treating patients with cholelithiasis. The recent introduction of agents that can safely dissolve gall stones and the advent of lithotripsy have added new dimensions to managing this common and economically important disorder. Gall bladder stones can be removed at operation, systemically dissolved with bile acids, crushed by extracorporeal shock wave, or locally dissolved with methyl tert-butyl ether; endoscopic sphincterotomy has been successful in managing common bile duct stones. Doctors can now offer several therapeutic options to patients with gall stones; and though this has enhanced the scope for manoeuvre, it has created new difficulties in making decisions.

\section{Diagnosis}

Gall stones may present in various ways. The most common and characteristic are either acute cholecystitis or obstruction of the common bile duct. Only $5 \%$ of patients with acute cholecystitis do not have coexistent gall stones. Thus stones may commonly rupture into the bowel, obstruct the terminal ileum, or cause pancreatitis. Gall stones and cancer of the gall bladder may coexist. A few patients with cholecystitis may have jaundice but not choledocholithiasis, but most patients with gall stones who are jaundiced have stones obstructing the commn bile duct. Cholangitis is diagnosed when, in addition to cholestasis, there are chills, fever, and pain and tenderness in the right upper quadrant. Flatulence, intolerance of fatty food, and vague right upper quadrant and epigastric pains are often ascribed to gall stones and chronic cholecystitis. Such complaints, however, are equally often related to other common gastrointestinal disorders, and one of the main difficulties for doctors is to decide what treatment to offer patients with gall stones who present with these symptoms alone. Because only $15 \%$ of gall stones are radio-opaque a plain abdominal radiograph is often unhelpful. Ultrasonography or oral cholecystography, or both, are the basic diagnostic tools. When choledo- cholithiasis is suspected endoscopic retrograde cholangiopancreatography or percutaneous transhepatic cholangiography can be performed in addition to ultrasonic scanning and computed tomography.

\section{Gall stones in the gall bladder}

Gall stones and acute cholecystitis are an indication for cholecystectomy, which is the major abdominal operation most often performed in Britain. The gall bladder is removed via a vertical paramedial incision or a subcostal incision, which is favoured in obese patients with a wide costal angle. Because choledocholithiasis occurs in $10-15 \%$ of patients, often with no overt symptoms, the common bile duct should also be investigated for gall stones. Until recently, operative cholangiography was considered to be essential in any patient undergoing cholecystectomy. The routine use of operative cholangiography has the advantage of reducing unnecessary bile duct exploration; of diagnosing gall stones that would otherwise have been overlooked by palpation, thereby reducing the number of residual gall stones in the common bile duct; and of showing unsuspected disease. Other techniques exist, however, for evaluating bile ducts, such as preoperative ultrasonography and peroperative choledocoscopy, which have the advantage of avoiding an operative cholangiogram.

The consensus has moved towards early operation for patients with acute cholecystitis. On admission to hospital they receive appropriate medical support. Analgesics recommended include morphine, pethadine, and pentazocine, the last having the theoretical advantage of not raising biliary pressure. Intravenous fluids are given if the patient is vomiting and an adequate urinary flow is maintained; it is seldom necessary to use a nasogastric tube. Appropriate treatment is provided for associated cardiac or pulmonary disease. There is no evidence that anticholinergic agents are of value.

The rational use of antibiotics in acute cholecystitis remains controversial. Only half the patients coming to operation have infected bile, from which various
Medinburgh EH3 9YW professor of medicine 